\title{
Large-Scale News Topic Tracking and Key-scene Ranking with Video Near-Duplicate Constraints
}

\begin{abstract}
To make full use of the overwhelming volume of news videos available today, it is necessary to track the development of news stories from different channels, mine their dependencies, and organize them in a semantic way. We propose a novel news topic tracking and re-ranking system. The main contributions include: (1) a novel scheme of mining topicrelated stories through tracking and re-ranking on the basis of near duplicates built on top of text, (2) a proposed simple but effective query-expansion algorithm for improving the representativeness of a search query, (3) a large-scale broadcast video database containing more than 34,000 news stories constructed for experimentation, and (4) a novel keyscene ranking scheme for analyzing both text similarity and video near-duplicate constraints.
\end{abstract}

\section{Categories and Subject Descriptors}

H.2.8 [Database Management]: Database ApplicationsData Mining; H.3.3 [Information Storage and Retrieval]: Information Search and Retrieval-Query Formulation, Search Process; I.2.7 [Artifical Intelligence]: Natural Language Processing - Text analysis

\section{General Terms}

Algorithms, Design, Experimentation, Performance

\section{Keywords}

News Topic Tracking, Key-scene Ranking, Near-Duplicate Detection, Video Data Mining

\section{INTRODUCTION}

Recent advances in broadband networks, storage devices, and digital video broadcasting have created a demand for large-scale broadcast video databases and intelligent access. Broadcast video, especially news video, contains a broad range of human activities, which could be considered as a

Permission to make digital or hard copies of all or part of this work for personal or classroom use is granted without fee provided that copies are not made or distributed for profit or commercial advantage and that copies bear this notice and the full citation on the first page. To copy otherwise, to republish, to post on servers or to redistribute to lists, requires prior specific permission and/or a fee.

WOODSTOCK' 97 El Paso, Texas USA

Copyright 200X ACM X-XXXXX-XX-X/XX/XX ...\$10.00. valuable cultural and social heritage. To make full use of the overwhelming volume of news videos available today, it is necessary to track the development of news stories from different channels, mine their dependencies, and organize them in a semantic way. Among these research efforts, topic tracking is a fundamental step for news browsing, retrieval, topic threading, and summarization. Topic tracking aims at linking together evolving and historical stories according to topics such as the Trial of Saddam Hussein and the 7 July 2005 London bombings.

\section{$1.1 \quad$ Topic Tracking}

Topic tracking is normally studied under the theme of Query by Example (QBE) with textual features as the underlying cues $[1,3,4,5,11]$. A QBE parser parses the search query, e.g., the text of a full document obtained from web pages or closed-caption of news videos, and looks for the keywords. Similar documents are searched for based on these keywords. However, textual information is not discriminating enough to distinguish documents of similar but irrelevant topics. Also, the keywords parsed from the search query are normally limited and not informative enough to represent the characteristics of the corresponding news topic.

In addition to text transcripts, news videos provide richer visual information. In news videos, there are a number of near duplicates that appear at different times and dates and across various broadcast sources. Near duplicates, by definition, are sets of shots composed of the same video material used several times in different programs or material involving the same scene. These materials are possibly different from each other in terms of video editing or camerawork, as shown in Fig. 1. These near duplicates basically form pairwise equivalent constraints that are useful for bridging evolving news stories across time and sources.

Duygulu et al. [2] presented a technique for mining and tracking the repeated sequence of shots based only on nearduplicate detection. This work assumes that the coverage, i.e. the percentage of stories that share near duplicates with other stories in the same topic, is sufficient enough for complete and thorough topic tracking. However, this assumption cannot be satisfied with large-scale databases. Zhai et al. [7] linked news stories by combining keyframe matching and textual correlation. Hsu et al. [8] tracked four topics with visual duplicates and semantic concepts, and found that near duplicates significantly improve tracking performance. These two works use near-duplicate and textual information as two independent modalities, and the potential semantic relatedness between them are not well explored and thus wasted. Different from these multi-modality fu- 


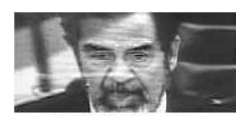

FNN SPEAK

2006/11/06

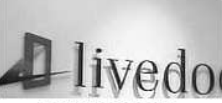

FNN SPEAK

2006/02/13

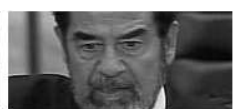

FNN SPEAK

$2006 / 12 / 27$

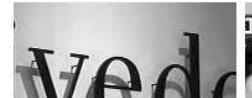

NHK NEWS 7

$2006 / 01 / 16$

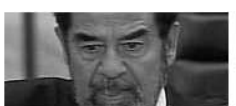

FNN SPEAK

$2006 / 12 / 28$
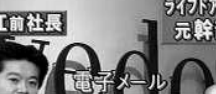

NHK NEWS 7

$2006 / 02 / 12$

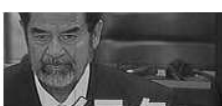

NHK NEWS 7

2006/11/05

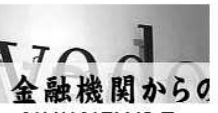

NHK NEWS 7

2006/03/13

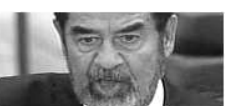

NHK NEWS 7

2006/12/27

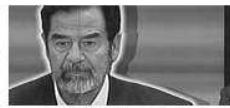

NHK NEWS 7

2006/12/29

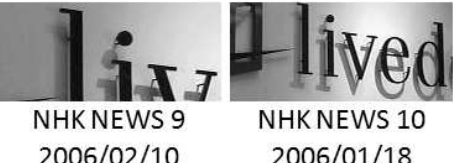

Figure 1: Near duplicates across different stories of two topics. The label under each keyframe is the program name and the airdate. Above: Trial of Saddam Hussein. Below: Fraud allegations of Livedoor.

sion studies, Wu et al. [12] presented a system built on visual near-duplicate constraints, which are applied on top of text to improve the story clustering and mining. This work depends on manual near-duplicate labeling, which is impossible to handle with large-scale databases.

We offer a new perspective by mining topic-related stories through tracking and re-ranking on the basis of near duplicates built on top of text. We propose a simple but effective query-expansion algorithm for improving the representativeness of a search query. A large-scale broadcast video database containing more than 34,000 news stories (compared to around 800 news stories used by Wu et al. [12]) was constructed for experimentation.

\subsection{Topic Summarization}

Providing summarization and visualization of large-scale news videos is an important task, which aims at saving time for general audiences in searching and reading news of interest. One solution for this task is to let the audience track up and down the topic thread structure originating from a specified story $[6,10]$. However, in a large-scale broadcast video database, there are tens or hundreds of news stories depicting the same topic. It is still a large burden for the audience to browse so many stories in one interface.

Mo et al. provided another solution for extracting important images from stories depicting the same topic [5]. In this work, near duplicates are detected using normalized cross correlation (NCC) between two frames. The frequency of near duplicates is analyzed for extracting important images. Considering the fact that both text-based topic tracking and near-duplicate detection are erroneous, the method proposed by Mo et al. [5] might generate a certain number of images irrelevant to the corresponding topic.

In this paper, news stories are tracked and re-ranked based on near-duplicate detection. Detected near-duplicate shots are defined as key-scenes. Both text similarity and video near-duplicate constraints are analyzed for evaluating the importance degree of these key-scenes, which is further used for key-scene ranking.

\section{FRAMEWORK OVERVIEW}

Our system works on a large-scale broadcast video database. Given a news story used as the search query, the system outputs stories depicting the same topic and important keyscenes representing this topic. Formally, a news story is defined as a semantic segment within a news video containing a report depicting a specific topic or incident. A story is described by a group of shots. Each shot is described by a set of representative keyframes and closed-captions. Figure 2 depicts our proposed news topic tracking and key-scene ranking system.

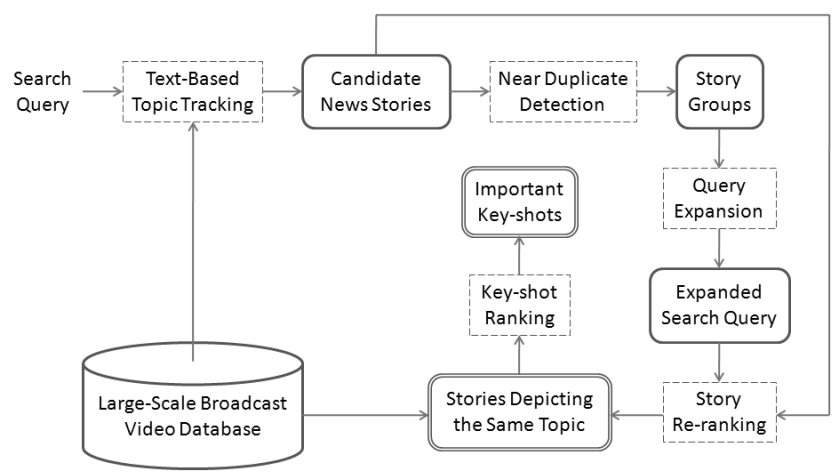

Figure 2: Proposed news topic tracking and keyscene ranking system

Initially, candidate news stories similar to the query are searched using a topic tracking method based only on textual information. The reason that we first use a text-based method before near-duplicate detection is because the latter requires processing of visual information and is computationally far more expensive than processing of textual information. On the other hand, the coverage of near duplicates is normally not sufficient enough for complete and thorough topic tracking compared to textual information. After textbased topic tracking, near duplicates are detected from the set of candidate news stories and used to group stories that share these near duplicates. A query-expansion algorithm is then used to improve the representativeness of a search query based on story groups. The expanded search query is used to re-rank news stories depicting the same topic within the database.

Finally, detected near-duplicate shots are defined as keyscenes. Both text similarity and video near-duplicate constraints are analyzed for evaluating the importance degree of these key-scenes. Key-scenes are further ranked based on their importance degree and used for representing the corresponding topic.

\section{NEWS TOPIC TRACKING}




\subsection{Text-Based Topic Tracking}

Topic tracking is normally studied under the theme of Query by Example (QBE) with textual features as the underlying cues $[1,3,4,5,11]$. In news videos, the focal point or content of evolving stories depicting the same topic normally varies slowly with time. The topic tracking method used in this work should be robust enough over this focalpoint variation. In this paper, an algorithm developed by Ide et al. [3] is used for this purpose, which uses semantic and chronological relation to track the chain of related news stories in the same topic along time. This procedure forms a simple story link tree, such as the structure in Fig. 3, starting from the story of interest, i.e. the search query. This link tree can be also considered as a set of candidate news stories similar to the search query, which is further used for near-duplicate detection.

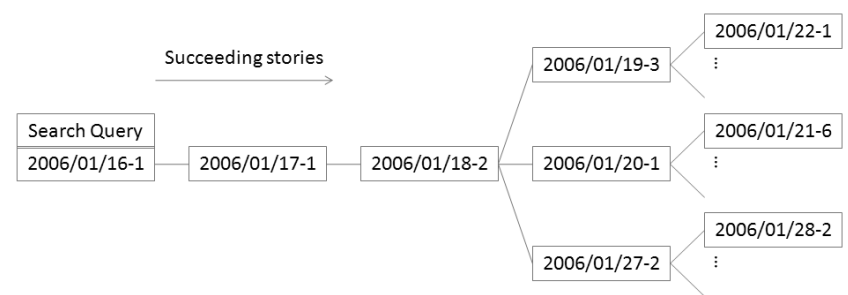

Figure 3: Example of simple story link structure

\subsection{Near-Duplicate Detection}

After text-based topic tracking, near duplicates are detected only from the set of candidate news stories. This can not only dramatically reduce the computation burden due to processing of visual information, but also reduce the probability of potential errors caused by near-duplicate detection. We used an interest-point-based algorithm with a local description for near-duplicate detection. This LIP-IS+OOS algorithm was proposed by $\mathrm{Ngo}$ et al. [9] and proved to be robust against variations of translation and scaling introduced due to video editing and different camerawork.

Ngo et al. tested their algorithm using a keyframe database instead of a video archive [9]. To extend LIP-IS+OOS to near-duplicate shot detection, we extract multiple keyframes from each video shot. The shot length is equally divided, and the frames at the points of division are selected as the keyframe. This is to tolerate the variation introduced due to camera and object motion. In equation terms, given the shot length $L$, the $(i \times L /(N+1))^{\text {th }}$ frames are extracted as the keyframe, with $i=1 \cdots N$. N indicates the number of keyframes and is empirically set to 3 in this paper.

In news videos, stories of different topics broadcasted from the same news program normally share similar video captions. As shown in Fig. 4 (1), the two keyframes are extracted from stories depicting two different topics but share the same time display (upper left), the same editing pattern (upper right), and similar video captions (below). From our experimental results, we also found a large number of false alarms sharing similar video captions detected as near duplicates. To tolerate this significant impact on the accuracy of near-duplicate detection, we propose to crop the keyframe beforehand. As shown in Fig. 4 (2), only the central part, where there are no video captions, is used for near-duplicate detection.
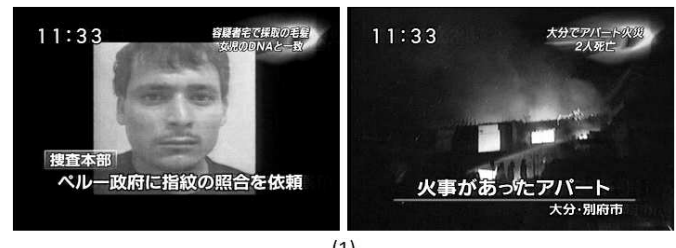

(1)

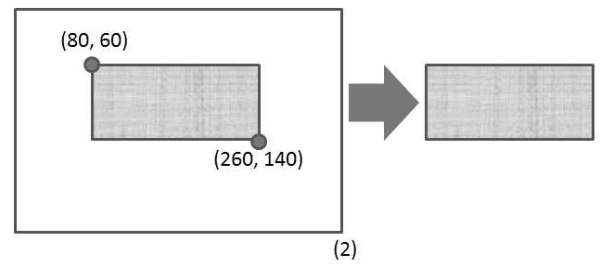

Figure 4: Keyframe cropping. (1) Example of keyframe pairs sharing similar video captions but different topics. (2) Crop central part for nearduplicate detection.

On the other hand, we also manually excluded anchorperson shots that are not related to the topic but are highly possible to be detected as near duplicates. Since anchorpersonshot detection has been extensively studied and many good algorithms were already proposed, this process can be automated if needed.

\subsection{Query Expansion}

\subsubsection{Story Grouping}

Given a story used as a search query, candidate stories similar to the query are searched across various news programs. Two stories are linked together if they share at least one pair of near-duplicate keyframes. Stories are then clustered into groups based on these links. Due to the potential errors caused by story segmentation and near-duplicate detection, two stories linked together might not be related to each other. We make the two following assumptions.

Assumption 1: Most stories in the same story group depict the same topic. This assumption is feasible in most cases because near duplicates are detected only from the set of candidate stories similar to the query so that the probability of potential errors caused by near duplicate detection is small.

Assumption 2: The largest story group depicts the same topic as the query. This assumption is also feasible because most near duplicates are shared between stories depicting the same topic as the query. From another point of view, the noise and outlier topics are normally different from each other so that few near duplicates are shared between them.

The feasibility of these two assumptions is also discussed in Section 4.2. Based on these assumptions, the largest story group is chosen as the expanded query to represent the characteristics of the corresponding news topic that the query depicts.

\subsubsection{Keyword Weighting}

One of the best known schemes for text mining is term frequency-inverse document frequency $(t f$-idf $)$ weighting. We 
propose to use a modified $t f$-idf weight and a keyword cooccurrence weight to describe the expanded query. To do so, semantic analysis is first applied to the compound nouns extracted from each story to generate a keyword vector for four semantic classes, general, personal, locational/organizational, and temporal. By observing our experimental results, we found that keywords of the temporal class are normally not helpful for identifying news stories depicting the same topic. Therefore, only the compound nouns of the other three classes are used as the keywords.

Different from traditional $t f-i d f$ weighting schemes, we consider one story group as one document. The story group is denoted as $\mathbb{S}$. Thus we have the term frequency $(t f)$, defined by Equation 1, where $n\left(t_{i}\right)$ denotes the number of occurrences of the considered keyword $t_{i}$ in the document. The denominator denotes the sum of occurrences of all keywords in the document.

$$
t f\left(t_{i}\right)=\frac{n\left(t_{i}\right)}{\sum_{t_{j} \in \mathbb{S}} n\left(t_{j}\right)}
$$

The inverse document frequency $(i d f)$ is a measure of the general importance of the keyword. We consider one story as one document, which is different from Equation 1. All news stories extracted from $2005 / 10 / 19$ to $2007 / 01 / 19$ were used to construct the document corpus for $i d f$ evaluation. The corpus is denoted as $\mathbb{C}$, and composed of more than 34,000 documents (denoted by $s$ ) broadcasted from six different news programs. Thus we have the $i d f$, defined by Equation 2, where $|\mathbb{C}|$ denotes the total number of documents in the corpus $\mathbb{C}$. The denominator denotes the number of documents where the keyword $t_{i}$ appears. The $t f-i d f$ is then defined by Equation 3.

$$
\begin{gathered}
i d f\left(t_{i}\right)=\log \frac{|\mathbb{C}|}{\left|s: t_{i} \in s, s \in \mathbb{C}\right|} \\
t f i d f\left(t_{i}\right)=t f\left(t_{i}\right) \times i d f\left(t_{i}\right)
\end{gathered}
$$

As an example, keywords with the six largest $t f$-idfs on the Trial of Saddam Hussein are listed in Table 1. We can see that our proposed keyword-weighting algorithm has removed unrelated or potentially ambiguous keywords, e.g., this goround, president Bush, United States, and November 5th, from the original query. On the other hand, keywords more related to the topic, e.g. Iraq and Shiah, are evaluated with higher importance.

For the keyword co-occurrence weight, we consider one story as one document and one story group as one document corpus. The co-occurrence frequency $(c f)$ of each keyword pair $\left(t_{i}, t_{j}\right)$ is first evaluated (Equation 4$)$. The numerator denotes the number of documents where both $t_{i}$ and $t_{j}$ appear in the document corpus $\mathbb{S}$. $|\mathbb{S}|$ denotes the total number of documents in $\mathbb{S}$. Thus, we have the keyword co-occurrence weight $(c w)$, defined by Equation 5 .

$$
\begin{gathered}
c f\left(t_{i}, t_{j}, \mathbb{S}\right)=\frac{\left|s: t_{i} \in s, t_{j} \in s, s \in \mathbb{S}\right|}{|\mathbb{S}|} \\
c w\left(t_{i}, t_{j}, \mathbb{S}\right)=c f\left(t_{i}, t_{j}, \mathbb{S}\right) \times i d f\left(t_{i}\right) \times i d f\left(t_{j}\right)
\end{gathered}
$$

For the expanded query or a single story (from Section 3.3.2), a keyword vector $\vec{V}$ can be created as follows.

$$
\vec{V}=\left(t f i d f\left(t_{1}\right), t f i d f\left(t_{2}\right), \cdots, t f i d f\left(t_{N}\right)\right)
$$

For expanded query $\mathbb{S}$, we use $\vec{V}_{\mathbb{S}}$ to denote the vector with $N$ in Equation 6 denoting the number of keywords in $\mathbb{S}$; in the case of a story $s, \vec{V}_{s}$ denotes the vector with $N$ denoting the number of keywords in this story. The keyword similarity between the expanded query and a story is thus defined by the cosine similarity shown in Equation 7 .

$$
r_{1}(\mathbb{S}, s)=\frac{\vec{V}_{\mathbb{S}} \cdot \vec{V}_{s}}{\left\|\vec{V}_{\mathbb{S}}\right\|\left\|\vec{V}_{s}\right\|}
$$

On the other hand, the keyword co-occurrence similarity between $\mathbb{S}$ and $s$ is defined by Equation 8. Finally, the text similarity between $\mathbb{S}$ and $s$ is defined as the weighted sum of $r_{1}$ and $r_{2}$ (Equation 9), with $w_{1} \in[0,1]$.

$$
\begin{gathered}
r_{2}(\mathbb{S}, s)=\frac{\sum_{t_{i} \in s, t_{j} \in s} c w\left(t_{i}, t_{j}, \mathbb{S}\right)}{\sum_{t_{i} \in \mathbb{S}, t_{j} \in \mathbb{S}} c w\left(t_{i}, t_{j}, \mathbb{S}\right)} \\
r(\mathbb{S}, s)=w_{1} \times r_{1}(\mathbb{S}, s)+\left(1-w_{1}\right) \times r_{2}(\mathbb{S}, s)
\end{gathered}
$$

\section{EXPERIMENTS}

\subsection{Database}

We tested our system with a large-scale broadcast video database comprised of actual broadcasted videos from 2005/10/19 to $2007 / 01 / 19$. These videos were broadcasted from six different news programs (FNN SPEAK, NHK NEWS 7, NHK NEWS 9, NHK NEWS 10, NNN NEWS DASH, NNN NEWS PLUS 1) produced by three different Japanese TV stations (Fuji Television, NHK, and Nippon Television). Closedcaptions were segmented into stories using the algorithm developed by Ide et al. [3], and videos were segmented into shots by comparing the RGB histograms between adjacent frames. Stories and shots were used as the basic units of analysis. The keywords were derived from a list of compound nouns extracted from closed-captions [3], while the keyframes were derived by equally dividing the shot and selecting the points of division. Note that each shot is represented by three keyframes. The set of near-duplicate keyframe pairs is detected using the algorithm developed by Ngo et al. [9]. The keyframes with the anchorperson has been removed. The database is comprised of 34,279 news stories.

Ten search queries are selected for experimentation, as listed in Table 2, including five Japanese and five foreign news. Candidate stories similar to these queries were searched across the six news programs, and near duplicates were detected from the set of candidate news stories. The duration within which the search was conducted varied from 1 to 15 months. Based on our proposed query-expansion algorithm, experiments on news story re-ranking were conducted. Without the official annotation of stories, we built a ground-truth table by manually labeling the result stories of the search according to topic themes.

\subsection{Experimental Results of Story Grouping}

\subsubsection{Text Similarity Based on Expanded Query}


Table 1: Keywords with six largest $t f$-idfs on Trial of Saddam Hussein

\begin{tabular}{|c|l|c|l|}
\hline \multicolumn{2}{||}{ Original Query } & \multicolumn{2}{|c|}{ Expanded Query } \\
\hline Rank & Keyword & Rank & Keyword \\
\hline 1 & sentence & 1 & former president Hussein \\
\hline 2 & this go-round & 2 & execution \\
\hline 3 & former president Hussein & 3 & capital punishment \\
\hline 4 & president Bush & 4 & Iraq \\
\hline 5 & United States & 5 & sentence \\
\hline 6 & November 5th & 6 & Shiah \\
\hline
\end{tabular}

Table 2: Ten search queries selected for experimentation

\begin{tabular}{|l|l|l|l|}
\hline Topic Number & Topic & Duration & Domestic / Foreign \\
\hline T1 & Trial of Saddam Hussein & 15 months & Foreign \\
\hline T2 & Architectural forgery in Japan & 2 months & Domestic \\
\hline T3 & Fraud allegations of Livedoor & 2 months & Domestic \\
\hline T4 & Trial of Saddam Hussein & 2 months & Foreign \\
\hline T5 & 7 July 2005 London bombings & 1 month & Foreign \\
\hline T6 & Murder of Airi Kinoshita & 1 month & Domestic \\
\hline T7 & Murder of Yuki Yoshida & 1 month & Domestic \\
\hline T8 & Murder of Goken Yaneyama & 1 month & Domestic \\
\hline T9 & 2006 North Korean missile test & 1 month & Foreign \\
\hline T10 & 2006 North Korean nuclear test & 1 month & Foreign \\
\hline
\end{tabular}

Experimental results of story grouping based on the ten search queries are listed in Table 3. From \#Story (\#TP), we can see that most news stories clustered in the same group depict the same topic as the search query except for T6. In other words, both Assumption 1 and Assumption 2 described in Section 3.3.1 were demonstrated to be feasible in our experiment. For T6, the news topic is on a child abduction-murder that occurred in Hiroshima, and the query story was broadcasted on 2005/12/01. The next day, another child abduction-murder was reported near Tokyo. In most news programs, these two news topics were broadcasted continuously. Due to the high similarity between them, the story segmentation method [3] used in this paper failed to segment them from each other, so that stories of the query topic also contain shots of the noise topic. As a result, stories depicting these two topics were clustered in the same story group based on near-duplicate detection.

\subsection{Story Re-ranking}

The aim of this section is to justify the performance of our proposed query-expansion algorithm. We evaluate the similarity between each news story tracked using the algorithm developed by Ide et al. [3] and the expanded query using the algorithm proposed in Section 3.3.3. In Equation 9, $w_{1}$ is empirically set to 0.5 . These stories are then ranked based on the computed similarity. Normally, stories depicting the same topic as the search query, i.e. relevant stories, should be associated with a higher rank than those depicting similar but irrelevant topics. To evaluate the performance, we compare our algorithm against a baseline algorithm used by Ide et al. [3]. In this baseline algorithm, the similarity between each news story and the original query is evaluated by adopting cosine similarity between two keyword frequency vectors generated from them. This similarity is then used for story re-ranking. An evaluation using average precision (AveP) was performed using Equation 10, and Fig. 5 illustrates the results. In Equation 10, $r$ denotes the rank, $N$ the number of stories searched, $\operatorname{rel}()$ a binary function on the relevance of a given rank, and $P()$ the precision at a given cut-off rank. $N_{r e l}$ denotes the number of relevant stories with $N_{r e l} \leq N$.

$$
\text { AveP }=\frac{\sum_{r=1}^{N}(P(r) \times \operatorname{rel}(r))}{N_{r e l}}
$$

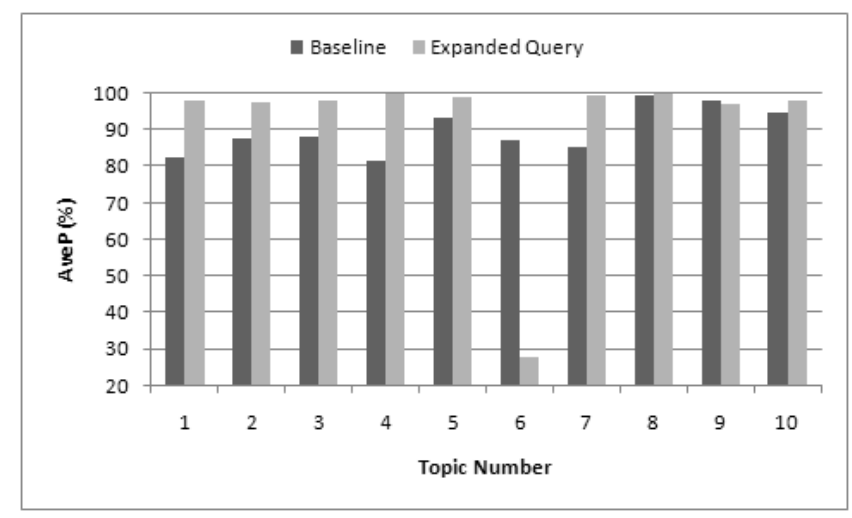

Figure 5: Average precision comparison for story re-ranking

From Fig. 5, we can see that the proposed query expansion algorithm outperformed the baseline for most topics except for T6 and T9. For T6, due to the reason explained in Section 4.2, a large number of stories depicting another different child abduction-murder were associated with higher ranks than relevant stories. T9 is the topic on the 2006 North Korean missile test broadcasted from 2006/07/06 to 
Table 3: Experimental results of story grouping. \#Candidate: number of candidate stories tracked by the algorithm developed by Ide et al. [3]. \#TP: number of relevant stories depicting the same topic as the query. \#Group: number of story groups constructed based on near-duplicate detection. \#Story: number of stories in the story group used for query expansion.

\begin{tabular}{|l|l|l|l|}
\hline Topic Number & \#Candidate (\#TP) & \#Group & \#Story (\#TP) \\
\hline T1 & $106(43)$ & 11 & $9(9)$ \\
\hline T2 & $370(174)$ & 3 & $72(72)$ \\
\hline T3 & $164(115)$ & 3 & $75(71)$ \\
\hline T4 & $35(13)$ & 3 & $10(10)$ \\
\hline T5 & $93(64)$ & 1 & $47(47)$ \\
\hline T6 & $148(25)$ & 7 & $28(4)$ \\
\hline T7 & $119(60)$ & 7 & $26(22)$ \\
\hline T8 & $35(34)$ & 3 & $13(13)$ \\
\hline T9 & $48(44)$ & 2 & $30(30)$ \\
\hline T10 & $65(54)$ & 3 & $39(38)$ \\
\hline
\end{tabular}

2006/08/06. The number of noise topics broadcasted during this period, which are different from but similar to the query topic, was small; therefore, both the baseline and our proposed query-expansion algorithm delivered high performance (98.28\% for baseline and $97.37 \%$ for expanded query).

\section{KEY-SCENE RANKING}

For this paper, we define detected near-duplicate shots as key-scenes. Two shots are linked together if they share at least one pair of near-duplicate keyframes. These shots are then clustered into groups based on these links. One group corresponds to one scene and is denoted as one key-scene. Due to the potential errors caused by text-based topic tracking and near-duplicate detection, these key-scenes normally contain a certain number of scenes irrelevant to the corresponding topic. In Section 4.3, the similarity $r(\mathbb{S}, s)$ between each news story $s$ and the expanded query $\mathbb{S}$ has been evaluated using the algorithm proposed in Section 3.3.3. This similarity is considered to be helpful for removing the irrelevant scenes described above and thus used as one of the cues for evaluating the importance degree of key-scenes.

On the other hand, the set of key-scenes also contains scenes of reporters that are not useful for representing the topic. Figure 6 shows some examples of important scenes as well as scenes of reporters. The number of programs across which the corresponding scene is broadcasted $n_{P R}$, number of stories across which the corresponding scene is broadcasted $n_{S T}$, and number of shots in the corresponding key-scene group $n_{S H}$ were evaluated for each scene. Note that $n_{P R} \leq n_{S T}$ and $n_{S T} \leq n_{S H}$ for all cases. From these examples, we can obtain the following observations.

Observation 1: Important scenes are normally broadcasted across different news programs (e.g. $n_{P R} \geq$ 2 ), while scenes of reporters are only used by one single program $\left(n_{P R}=1\right)$.

Observation 2: Within one story, scenes of reporters are normally used more frequently than important scenes (i.e. scenes of reporters tend to have lower value of $n_{S T} / n_{S H}$ than important scenes).

Based on the discussion and observations described above, we propose to use three types of statistics for key-scene ranking. Given a key-scene $k_{i}$, The first statistic $s r_{P R}\left(k_{i}\right)$ indicates the ratio of $n_{P R}\left(k_{i}\right)$ over the number of all news programs $N_{P R}$ (Equation 11). Generally, key-scenes with higher $s r_{P R}$ are determined as more important scenes.

$$
s r_{P R}\left(k_{i}\right)=\frac{n_{P R}\left(k_{i}\right)}{N_{P R}}
$$

The second statistic $s r_{S T}\left(k_{i}\right)$ indicates the ratio of $n_{S T}\left(k_{i}\right)$ over $n_{S H}\left(k_{i}\right)$ (Equation 12). $s r_{S T}\left(k_{i}\right)$ can also be considered as the inverse of the average frequency of using the corresponding scene within one news story. Based on Observation 2, key-scenes with higher $s r_{S T}$ are determined as more important scenes.

$$
s r_{S T}\left(k_{i}\right)=\frac{n_{S T}\left(k_{i}\right)}{n_{S H}\left(k_{i}\right)}
$$

For the third statistic, we assume that key-scenes broadcasted across important stories are also important. Given $s$ denoting the story, the group of stories across which the keyscene $k_{i}$ is broadcasted can be denoted by Equation 13 with $\left|\mathbb{G}_{i}\right|=n_{S T}\left(k_{i}\right)$. Thus, we have the third statistic $s r_{R}\left(k_{i}\right)$, defined by Equation 14. Here, $\mathbb{S}$ denotes the expanded query, and $r(\mathbb{S}, s)$ denotes the similarity between each news story and the expanded query (see Section 3.3.3 for reference). Key-scenes with higher $s r_{R}$ are determined as more important scenes.

$$
\begin{gathered}
\mathbb{G}_{i}=\left\{s: k_{i} \in s\right\} \\
s r_{R}\left(k_{i}\right)=\frac{\sum_{s \in \mathbb{G}_{i}} r(\mathbb{S}, s)}{n_{S T}\left(k_{i}\right)}
\end{gathered}
$$

Finally, the importance degree of the key-scene $k_{i}$ is defined by Equation 15 with $w_{2} \in[0,1]$. Empirically, we recommend to set $w_{2}>0.5$. Figure 7 shows some examples of key-scenes ranked by $\operatorname{sr}\left(k_{i}\right)$ with $w_{2}=0.75$.

$$
s r\left(k_{i}\right)=\left(w_{2} \times s r_{P R}\left(k_{i}\right)+\left(1-w_{2}\right) \times s r_{S T}\left(k_{i}\right)\right) \times s r_{R}\left(k_{i}\right)
$$

Figure 7 (1) shows ten key-scenes extracted from T4 (Trial of Saddam Hussein). In this example, scenes of Iraqi Prime Minister Nouri al-Maliki, Saddam Hussein, and his capital punishment sentence are ranked with a higher importance degree (1a). The remaining five key-scenes with lower $\operatorname{sr}\left(k_{i}\right)$ shown in (1b) are all irrelevant scenes or reporter scenes. 

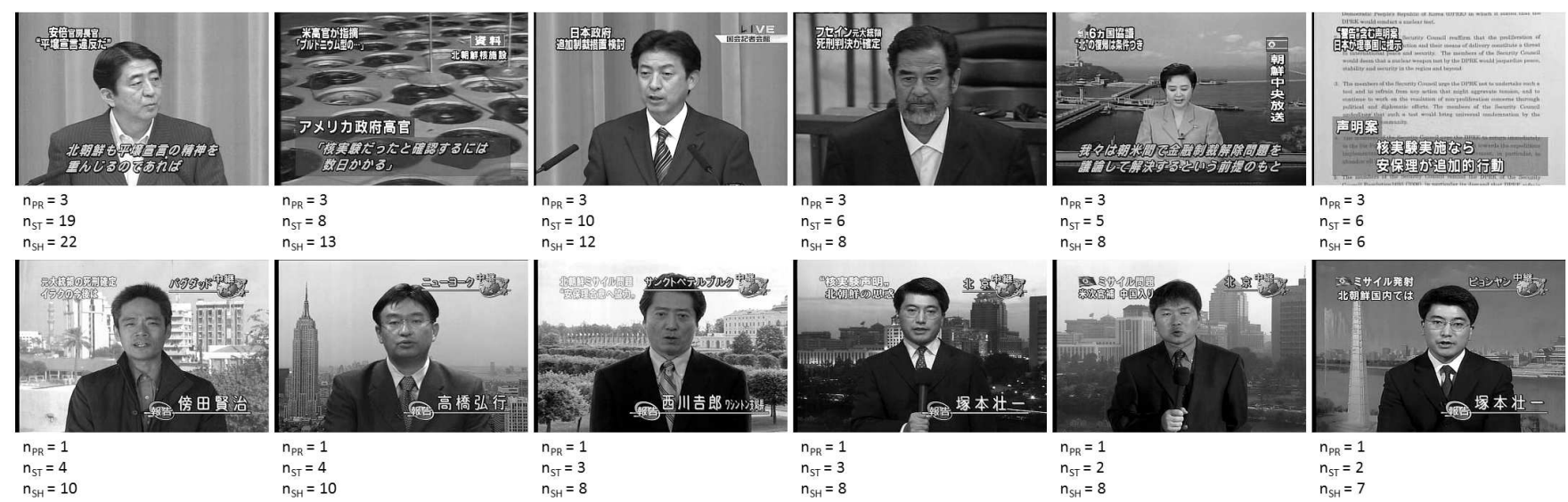

Figure 6: Examples of important scenes and scenes of reporters. $n_{P R}$ : number of programs across which corresponding scene is broadcasted. $n_{S T}$ : number of stories across which corresponding scene is broadcasted. $n_{S H}$ : number of shots in corresponding key-scene group. Above: important scenes. Below: scenes of reporters.

Note that in the least important key-scene in (1b), US Secretary of Defense Rumsfeld is talking on the legitimacy of the Iraq War, which is not directly related to Saddam Hussein. Therefore, the similarity between this story and the expanded query of T4 is low, which further decreased $s r_{R}$ used in Equation 15. In another example (Fig. 7 (2)), the topic class consisted of 59 key-scenes. Because we used Japanese news programs for experimentation, we found that a large number of scenes reflecting the attitude of the Japanese government against North Korea's nuclear test were frequently used in the videos. Therefore, a higher importance degree is associated to these scenes.

\section{CONCLUSIONS}

We proposed a novel news topic tracking and key-scene ranking system. This system offers a new perspective by exploring the potential semantic relatedness derived between near-duplicate and textual information. A large-scale broadcast video database containing more than 34,000 news stories (compared to around 800 news stories used by $\mathrm{Wu}$ et al. [12]) was constructed for experimentation. The experimental results showed that our proposed query-expansion algorithm based on near-duplicate detection outperformed traditional QBE methods that only use textual features as the underlying cues. A novel key-scene ranking scheme was also proposed by analyzing both text similarity and video near-duplicate constraints. This scheme is useful for visualizing the semantic structure of news topics as well as topicbased video indexing and retrieval.

As a future work, we are planning to conduct a comparison between our proposed query-expansion algorithm with other techniques, e.g. PageRank. We also plan to work on graphical visualization of news topics by integrating keyscene ranking with news-topic threading for practical application of our proposed system.

\section{REFERENCES}

[1] Chieu, H.L., Lee, Y.K.: Query based event extraction along a timeline. In: SIGIR, pp. 425-432 (2004)

[2] Duygulu, P., Pan, J.-Y., Forsyth, D.A.: Towards auto-documentary: tracking the evolution of news stories. In: ACM Multimedia, pp. 820-827 (2004)

[3] Ide, I., Mo, H., Katayama, N., Satoh, S.: Topic Threading for Structuring a Large-Scale News Video Archive. In: CIVR, pp. 123-131 (2004)

[4] Kumar, R., Mahadevan, U., Sivakumar, D.: A graph-theoretic approach to extract storylines from search results. In: Proceedings of the tenth ACM SIGKDD international conference on Knowledge discovery and data mining, pp. 216-225 (2004)

[5] Mo, H., Yamagishi, F., Ide, I., Katayama, N., Satoh, S., Sakauchi, M.: Key Image Extraction from a News Video Archive for Visualizing Its Semantic Structure. In: PCM (1), pp. 650-667 (2004)

[6] Ide, I., Kinoshita, T., Mo, H., Katayama, N., Satoh, S.: trackThem: Exploring a Large-Scale News Video Archive by Tracking Human Relations. In: AIRS, pp. 510-515 (2005)

[7] Zhai, Y., Shah, M.: Tracking news stories across different sources. In: ACM Multimedia, pp. 2-10 (2005)

[8] Hsu, W.H., Chang, S.-F.: Topic Tracking Across Broadcast News Videos with Visual Duplicates and Semantic Concepts. In: ICIP, pp. 141-144 (2006)

[9] Ngo, C.-W., Zhao, W., Jiang, Y.-G.: Fast tracking of near-duplicate keyframes in broadcast domain with transitivity propagation. In: ACM Multimedia, pp. 845-854 (2006)

[10] Ide, I., Kinoshita, T., Takahashi, T., Satoh, S., Murase, H.: mediaWalker: a video archive explorer based on time-series semantic structure. In: ACM Multimedia, pp. 162-163 (2007)

[11] Lin, F., Liang, C.-H.: Storyline-based summarization for news topic retrospection. Decis. Support Syst. 45, 473-490 (2008)

[12] Wu, X., Ngo, C.-W., Hauptmann, A.G.: Multimodal News Story Clustering With Pairwise Visual Near-Duplicate Constraint. IEEE Transactions on Multimedia. 10, 188-199 (2008) 


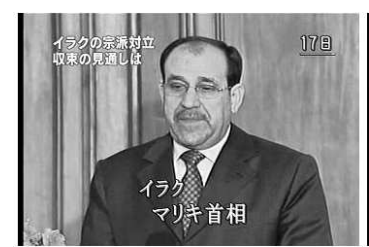

Rank: $1 / 10$

$\mathrm{sr}=0.32$

Iraqi prime minister Nouri al-

Maliki talking on trial of Saddam

Hussein

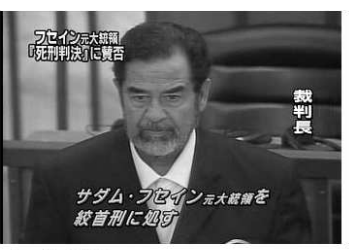

Rank: $2 / 10$

$\mathrm{sr}=0.31$

Saddam Hussein inside the

courtroom

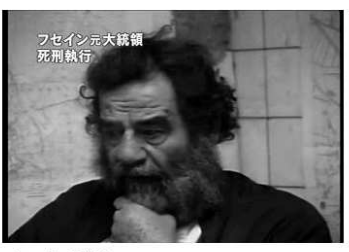

Rank: $3 / 10$

$\mathrm{sr}=0.29$

Saddam Hussein inside the prison

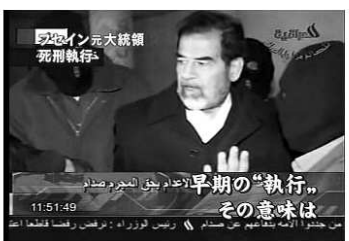

Rank: $4 / 10$

$\mathrm{sr}=0.29$

File footage of Saddam Hussein

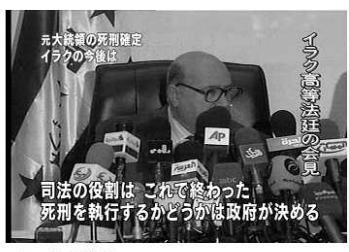

Rank: 5/10

$\mathrm{sr}=0.28$

Capital punishment sentence of

Saddam Hussein

(1a) 5 key-scenes with higher sr $\left(k_{i}\right)$ on T4

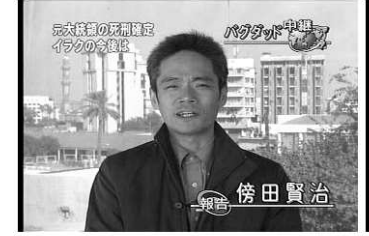

Rank: 6/10

$\mathrm{sr}=0.24$

Reporter scene

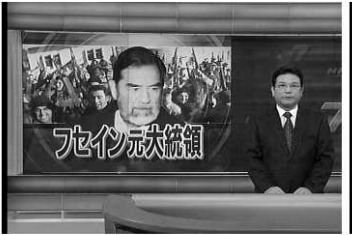

Rank: $7 / 10$

$\mathrm{sr}=0.23$

Reporter scene

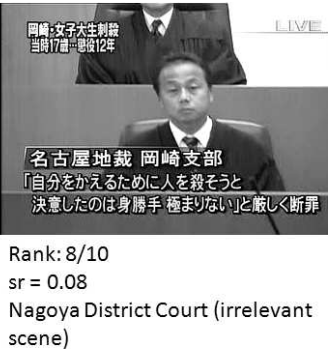

(1b) 5 key-scenes with lower $\operatorname{sr}\left(k_{i}\right)$ on T

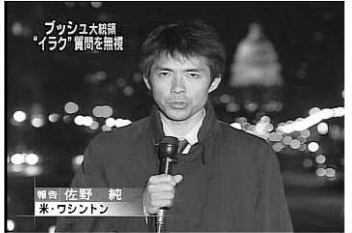

Rank: 9/10

$\mathrm{sr}=0.04$

Reporterscene

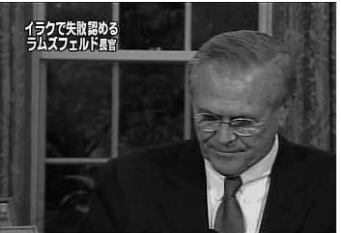

Rank: $10 / 10$

$\mathrm{sr}=0.02$

US Secretary of Defense Rumsfeld

talking on Iraq War (irrelevant scene)

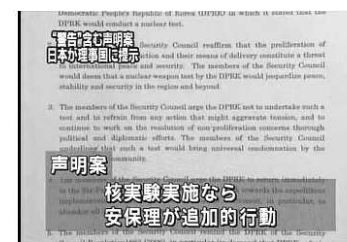

Rank: $1 / 59$

$\mathrm{sr}=0.52$

Japanese communique against

North Korea

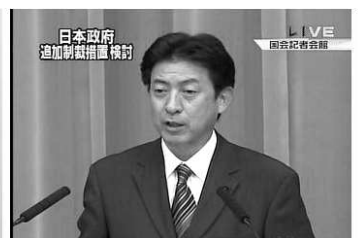

Rank: $2 / 59$

$s r=0.46$

Japanese chief cabinetsecretary talking on additional sanctions against North Korea

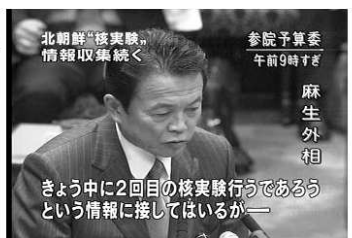

Rank: $3 / 59$

$\mathrm{sr}=0.43$

Japanese foreign minister talking on North Korea nuclear test

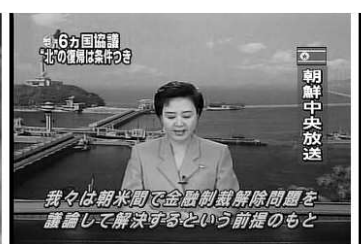

Rank: $5 / 59$

$s r=0.41$

North Korean news on returning to six-party talks

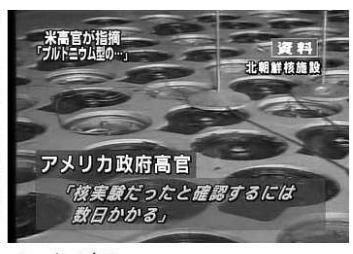

Rank: 8/59

$\mathrm{sr}=0.38$

Nuclearfacilities in North Korea

(2a) 5 key-scenes with higher $\operatorname{sr}\left(k_{i}\right)$ on T10

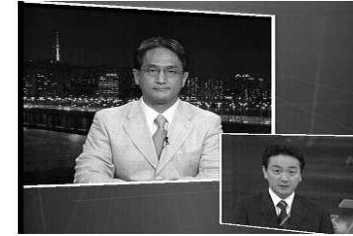

Rank: 40/59

$\mathrm{sr}=0.20$

Reporter scene

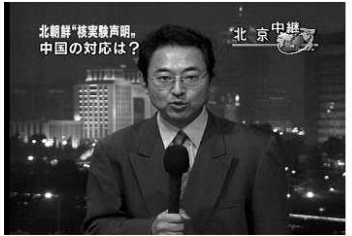

Rank: $45 / 59$

$\mathrm{sr}=0.17$

Reporterscene

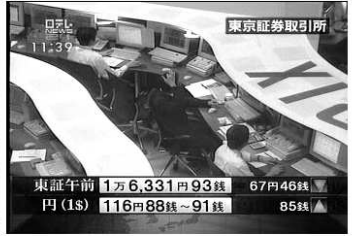

Rank: 53/59

$\mathrm{sr}=0.13$

TokyoStock Exchange (irrelevant scene)

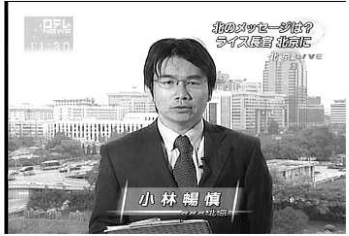

Rank: 54/59

$\mathrm{sr}=0.12$

Reporter scene

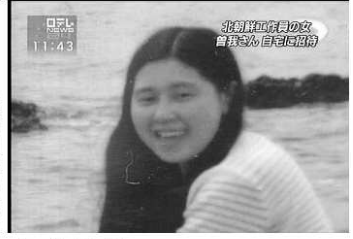

Rank: 59/59

$\mathrm{sr}=0.03$

North Korean agent (irrelevant scene)

(2b) 5 key-scenes with lower sr $\left(k_{i}\right)$ on T10

Figure 7: Examples of key-scenes ranked by $\operatorname{sr}\left(k_{i}\right)$. Rank indicates the rank number over the total number of key-scenes (given $n$ denoting the total number of key-scenes, $1 / n$ indicates the most important key-scene while $n / n$ indicates the lest important key-scene). $s r$ indicates the important degree defined by Equation 15 . The descriptions under $s r$ show the content of each key-scene. 